\title{
Beat-by-beat vascular responses during anticipatory heart rate deceleration
}

\author{
J. RICHARD JENNINGS \\ University of Pittsburgh, Pittsburgh, Pennsylvania
}

\begin{abstract}
The slowing of heart rate prior to an anticipated action is puzzling. Increased heart rate would seem biologically more appropriate. Beat-by-beat vascular measures were studied in order to see if heart rate slowing was associated with improved blood flow to the limb involved in the anticipated action. In 27 men, pulse wave velocity (PWV), pulse transit time (PTT), and pulse amplitude measures were collected from arterial and thumb sites. A paced auditory reaction time (RT) task with two levels of reward (incentive) was used. Beat-by-beat results showed a concomitance of heart rate slowing, PTT shortening, and pulse amplitude increase. These changes were interpreted as being due to vagal effects and their hemodynamic consequences. Pacing RT at fast speeds led to an elevated PWV, which suggested alpha sympathetic activation during periods requiring substantial effort. Tentatively, the results suggest that heart rate slowing is one aspect of an adaptive cardiovascular adjustment by joint vagal and alpha sympathetic activation.
\end{abstract}

Heart rate slows just prior to the response in a fixed-foreperiod reaction time (RT) task (e.g., J. I. Lacey, 1972). From a teleological perspective, this response is paradoxical-just prior to an event requiring exertion, the pumping rate providing support for that exertion is reduced. Furthermore, at least one other response, skin conductance, increases concomitantly with heart rate slowing (J. I. Lacey, Kagan, B. C. Lacey, \& Moss, 1963). Interpretively, sympathetically controlled skin conductance is increasing while heart rate is decreasing due to parasympathetic activation (Obrist, Webb, Sutterer, \& Howard, 1970). Why does heart rate slow when other responses have been activated via sympathetic efferents?

At least three conceptual solutions to the paradox have been offered. These solutions suggest that deceleration is adaptive because it facilitates preparation of the central nervous system, the motor system, or the vasicular system. The Laceys (e.g., B. C. Lacey \& J. I. Lacey, 1974, 1978) have suggested that the slowing of heart rate inhibits baroreceptor firing, which in turn permits the central nervous system to more effectively integrate stimulus and response. Obrist (e.g., Obrist, 1981; Obrist, Howard, Lawler, Galosy, Meyers, \& Gaebelein, 1974) has suggested that heart rate slows concomitantly with a motoric quieting. The quieting reduces "neural

I would like to thank Michael Sunderman and Sam Choi for their excellent assistance in collecting and analyzing the data. In addition, Michael Sunderman and Kay Jennings read an initial draft of this paper. The author's address is: Western Psychiatric Institute and Clinic, University of Pittsburgh, 3811 O'Hara Street, Pittsburgh, Pennsylvania 15213. noise" and thus facilitates behavior. Finally, we and others have considered the possibility of vascular effects (Jennings, Schrot, \& Wood, 1980; Williams, Bittker, Buchsbaum, \& Wynne, 1975). Since blood flow to the responding muscles is a more immediate support for action than are brief changes in heart rate, perhaps blood flow (cardiac output) or regional blood flow is facilitated by brief heart rate slowing. Two possible mechanisms might be noted. First, cardiac output might be enhanced by increased filling of the heart due to slow heart rate. Slow heart rate will lead to more time for the heart to be filled with blood. If sympathetic contractile effects were maintained, the result could be more blood being distributed at equivalent force relative to less volume distributed at a higher heart rate (Burton, 1972). A second mechanism might be cholinergically mediated changes in regional blood flow. Djojosugito, Folkow, Kylstra, Lisander, and Tuttle (1970) suggested that activation of the hypothalamic defense reflex produced maximal muscle blood flow if it occurred during baroreceptor activation (cf. Coote, Hilton, \& Perez-Gonzalez, 1979). The Laceys (e.8., Lacey, 1972; B. C. Lacey \& J. I. Lacey, 1978) have related anticipatory deceleration to baroreceptor mechanisms. Thus, anticipatory deceleration may be an indirect means of maximizing muscle perfusion. Although speculative, these thoughts led us to consider vascular changes concomitant with anticipatory cardiac deceleration. Should vascular changes be found, subsequent work would have to address the relative importance of baroreceptor, motoric, and vascular factors.

The current work follows up our initial report on vascular changes during choice RT (Jennings, Schrot, \& Wood, 1980). In that report, we examined 
vascular responses during a speed-accuracy tradeoff task and varied monetary incentive. The speedaccuracy tradeoff task paces choice RTs at different rates (producing a trading between speed and accuracy), and, more importantly, varies the amount of anticipatory cardiac deceleration. Varying incentives were employed to manipulate task involvement and perhaps level of tonic sympathetic activation during the task (e.g., Light \& Obrist, 1980; Manuck $\&$ Garland, 1979). The results suggested that incentive acted tonically to increase sympathetic outflow, while sympathetic activity was phasically inhibited to different degrees by the speed-emphasis conditions of the tradeoff task.

The current work employs the same paradigm but provides a more comprehensive assessment of beatby-beat vascular activity and of the effect of incentive. In the previous study, uncalibrated vascular measures were available from arterial and thumb sites only for the heartbeat during which the RT occurred. In this study, I report data for this beat and for the three beats before and the four beats after. Using subclavian and thumb sites, I was able to report arterial-to-arteriolar pulse wave velocity (PWV) in a single arterial tree. In addition, the measurement devices were calibrated to yield potentially more accurate amplitude measures (Jennings, Tahmoush, \& Redmond, 1980). Incentive was varied between volunteers in the previous work. A withinvolunteer design was employed in this study in hopes of providing a more sensitive assessment.

\section{METHOD}

\section{Volunteers}

Thirty-one young (mean age $=23$ years) men were paid volunteers. The volunteers were assigned randomly to one of two groups defined by the order of incentive conditions. Technical recording failures reduced the $\mathbf{n}$ to 27 for vascular data.

\section{Procedure and Task}

A speed-accuracy tradeoff paradigm was used. Wood and Jennings (1976) have provided a full description of this paradigm and the rationale for its use. In accordance with this paradigm, a choice RT task was performed, resulting in payoffs for varying response speeds; a $1,900-$ or $1,420-\mathrm{Hz}$ tone (at $62 \mathrm{dBA}$ ) signaled the press of a digit of the right or left hand on a response key. A right-hand response to the $1,420-\mathrm{Hz}$ tone and a left-hand response to the $1,900-\mathrm{Hz}$ tone were correct. Tones were $80 \mathrm{msec}$ in length. For a block of 70 trials, the volunteers were paid for correct responses within a given 100-msec time range (e.g., 100 to $200 \mathrm{msec}$ ). These time ranges were termed RT targets and were defined by the midpoints of the range at $150,200,250,300$, and $350 \mathrm{msec}$. In each experimental session, the volunteers received a block of 70 trials at each of the RT targets. The order of the target blocks was randomized. The tone occurred on the $\mathbf{R}$ wave of the first heartbeat following an 8-sec interstimulus interval. A feedback panel placed approximately $1 \mathrm{~m}$ in front of the volunteer was illuminated just after the third heartbeat following the stimulus. This panel indicated whether the correct response key had been pressed and whether the speed RT was too fast, too slow, or in the required range.
The volunteers participated in three 2-h sessions on separate days. First, the volunteers were trained in the speed-accuracy task during a practice day. In addition, a physiological baseline was collected and an initial block of RT trials was run with no feedback and with instructions to perform as fast and as accurately as possible. This block of trials assessed performance prior to the specific manipulation of reaction speed. Following this block, the volunteers were trained to vary response speed as required by the task. After the training day, the volunteers were assigned randomly to one of two orders of presentation of incentive conditions.

The low-incentive condition was defined by a $\$ .02$ reward for each correct response within the speed target window. The high-incentive condition was administered on a separate day and rewarded \$.05 for each correct, on-target response but penalized the volunteer $\$ .02$ for all other responses. In both conditions, the volunteers were informed after each block (target value) of the amount earned.

Each day was concluded by a 5-min resting physiological baseline and a postsession questionnaire. The questionnaire asked the volunteer to order the targets by degree of effort required and emotional involvement, to report the number of positive and negative emotions, and to note any strategies employed to improve performance.

Physiological messures. Measurements of heart rate, peripheral vascular activity, blood pressure, and breathing were used to assess cardiovascular reactivity. Heart rate was measured as interbeat intervals between $\mathbf{R}$ waves of the electrocardiogram. Beckman biopotential electrodes with paste were attached over the sternum, the left lateral margin of the rib cage, and the calf of the right leg. Resulting $\mathbf{R}$ waves were transformed into computer-compatible signals using a peak detector (Shimizu, 1978).

Time between transformed $\mathbf{R}$ waves was then measured to the nearest millisecond with the computer clock. Pretask interbeat interval (IBI) averages were computed during the presentation of sample tones, and posttask averages were computed from the resting baseline. During the "fast and accurate as possible" trials and the speed target blocks for the low- and high-incentive days, IBIs were collected for the three beats before the RT stimulus, the beat of the stimulus, and the four beats afterward.

Peripheral vascular activity was measured with two photometric devices calibrated in microwatt units according to the procedure described in Jennings, Tahmoush, and Redmond (1980). One device was placed on the thumbnail of the left hand, and the other was placed over the subclavian artery at the base of the neck. Attachment procedures have been described previously (Jennings, Tahmoush, \& Redmond, 1980). The distance between photo devices was measured so that PWV measures could be computed.

Blood pressure was measured with a surface Doppler probe placed next to a standard arm cuff. The cuff was inflated from the outside of the chamber. Cuff pressure and Doppler detections were recorded on the polygraph. Pressures were taken just prior to the end of each period in the experiment. For example, systolic and diastolic blood pressure from the 250-msec target block was collected immediately after the last RT but before the volunteers had been told the block was completed. Pressures were read by hand from the polygraph record.

Respiration was measured using a Yellow-Springs thermistor placed at the opening of the nares. This measure was used only to verify that major respiratory maneuvers, for example, breath holding, were not a consistent response to the task.

Equipment. The experimental task was controlled by a MINC (DEC) computer that also collected RT and IBI data on-line. The computer controlled auditory frequency through a HewlettPackard Model 3300D function generator and gated the auditory tone on, using a Hewlett-Packard Model 3302A trigger/ phase lock and a General Radio 1396B tone-burst generator. A General Radio Model 1390B was the white-noise generator. 
Tones and noise were presented over earphones. Closure of the microswitch response keys was sensed by the computer to measure RT from tone onset. The computer also controlled the LED response panel that signaled speed and correctness of response.

A Grass Model 7 polygraph provided signal conditioning. Laboratory-built devices were also used to condition the photometric and respiration signals prior to amplification by the polygraph. A Roche Arteriosonde 1020 provided the Doppler measure of blood pressure. All procedures were performed in an acoustic chamber with temperature control.

Data reduction. All physiological data were recorded on analog tape and reduced with the use of computer techniques. Analyses were performed on the IBIs for the three beats preceding the stimulus, the beat of the stimulus occurrence, and the four beats following the stimulus. With respect to the first beat, analyzed as Beat 1 , the stimulus occurred on the $R$ wave that ended Beat 3 and began Beat 4 .

A computer-detection procedure was used to measure photo signals for the same beats of the heart. The signals from both sites were sampled at a $1 / \mathrm{msec}$ rate for a $400-\mathrm{msec}$ interval following a 50-msec delay from the R wave of the EKG. The detection procedure located the maximum amplitude of the signal and worked backwards to find the maximum slope and onset of the systolic upstroke. Onset was identified when 10 consecutive points were found with a slope of less than two A/D units. The first point proximal to the upstroke (of the 10 points) was then defined as onset. The following photo-dependent measures were used: PWV computed between subclavian and thumb onset times (distance between sites expressed in meters divided by elapsed time expressed in seconds); time from $\mathbf{R}$ wave to subclavian onset; time from $\mathbf{R}$ wave to thumb onset; and amplitude (peak amplitude minus onset amplitude) for the thumb and subclavian signals.

Our attempt to score every relevant beat for vascular measures was only partially successful. Photo devices are susceptible to movement artifacts as well as to electronic noise. In addition, the computer algorithm was fallible. For these reasons, strict data editing based both on obtained distributions and knowledge of the polygraph output was employed. Data were retained only when both photo devices produced acceptable signals. The data remaining averaged about 1,750 beats per volunteer, although the $\mathrm{n}$ was only $35 \%$ of the total beats available. As might be expected, missing values were more likely to occur on beats associated with the RT response than on other beats. An analytic/ methodological strategy was used to overcome this problem. The task required responding from both hands on separate trials. Trials with the transducer on the responding hand could then be compared with trials with the transducer on the nonresponding hand. Results due to artifact would then appear only in the responding-hand data. Thus, artifactual findings were avoided by interpreting only those findings that were at least as strong in the nonresponding as in the responding hand.

\section{RESULTS}

\section{Performance}

The experiment manipulated RT target and payoffs in an effort to change motivational involvement and intensity of reaction. The performance results indicated that the manipulations had been effective. RT and proportion correct were assessed in a twoway repeated-measures analysis of variance, with incentive and RT targets as factors. A .05 rejection level was used in all analyses, and epsilon corrections were employed to correct degrees of freedom for repeated-measures factors (Jennings \& Wood, 1976).

Table 1 presents the mean RTs and proportion correct for the incentive and target conditions. Both measures were clearly manipulated by target condition [target main effect: $R T, F(1,28)=203.2$; proportion correct, $F(1,28)=161.5]$. Incentive condition, however, produced only marginal changes in RT $[F(1,28)=3.4, p<.1]$ and no significant change in proportion correct. Incentive condition did change the number of correct and appropriately timed responses, that is, rewarded responses, from 22.5 for the low-incentive conditions to 22.9 for the highincentive conditions $[F(1,24)=5.68]$. Thus, both $R T$ target and incentive conditions seemed to be effective, although RT target controlled performance much more than did incentive.

\section{Physiological Responses}

The physiological results will be topically organized around the experimental questions. Vascular, cardiac, and blood pressure results will be presented together, although each was analyzed separately using a repeated-measures analysis with factors of RT target, incentive, and, when possible, beat. Beatassessed changes related to the sequence of beats before, during, and after performance.

Beat-by-beat effects. A main question was whether beat-by-beat changes occurred in vascular as well as in IBI data. As in previous work, I obtained a clear lengthening of IBI prior to the stimulus that was followed by an accelerative recovery [beat main effect, $F(1,29)=44.0$; see Figure 1].

Were PWV and other vascular parameters also influenced by task performance on a beat-by-beat basis? PWV velocity was not, but PTT and pulse amplitude were. Figure 1 presents the beat-by-beat results for subclavian PTT and amplitude, along with the concomitant changes in IBI. PTT shortened at the time of stimulus presentation, and amplitude increased [beat main effect for PTT, $F(1,25)=12.9$; amplitude, $F(1,25)=6.2$, adjusted df]. Essentially the

Table 1

Reaction Time (in Milliseconds) and. Proportion Correct (PC) as a Function of Incentive and Target Conditions $(\mathrm{N}=31)$

\begin{tabular}{|c|c|c|c|c|c|c|c|c|c|c|}
\hline & \multicolumn{10}{|c|}{ Target } \\
\hline & \multicolumn{2}{|c|}{150} & \multicolumn{2}{|c|}{200} & \multicolumn{2}{|c|}{250} & \multicolumn{2}{|c|}{300} & \multicolumn{2}{|c|}{350} \\
\hline & RT & PC & $\mathrm{RT}$ & PC & RT & PC & $\mathrm{RT}$ & $\mathrm{PC}$ & RT & PC \\
\hline $\begin{array}{l}\text { Low Incentive } \\
\text { High Incentive }\end{array}$ & $\begin{array}{l}232 \\
227\end{array}$ & $\begin{array}{l}.50 \\
.50\end{array}$ & $\begin{array}{l}246 \\
240\end{array}$ & $\begin{array}{l}.55 \\
.53\end{array}$ & $\begin{array}{l}279 \\
273\end{array}$ & $\begin{array}{l}.63 \\
.63\end{array}$ & $\begin{array}{l}341 \\
342\end{array}$ & $\begin{array}{l}.79 \\
.82\end{array}$ & $\begin{array}{l}377 \\
370\end{array}$ & $\begin{array}{l}.90 \\
.89\end{array}$ \\
\hline
\end{tabular}




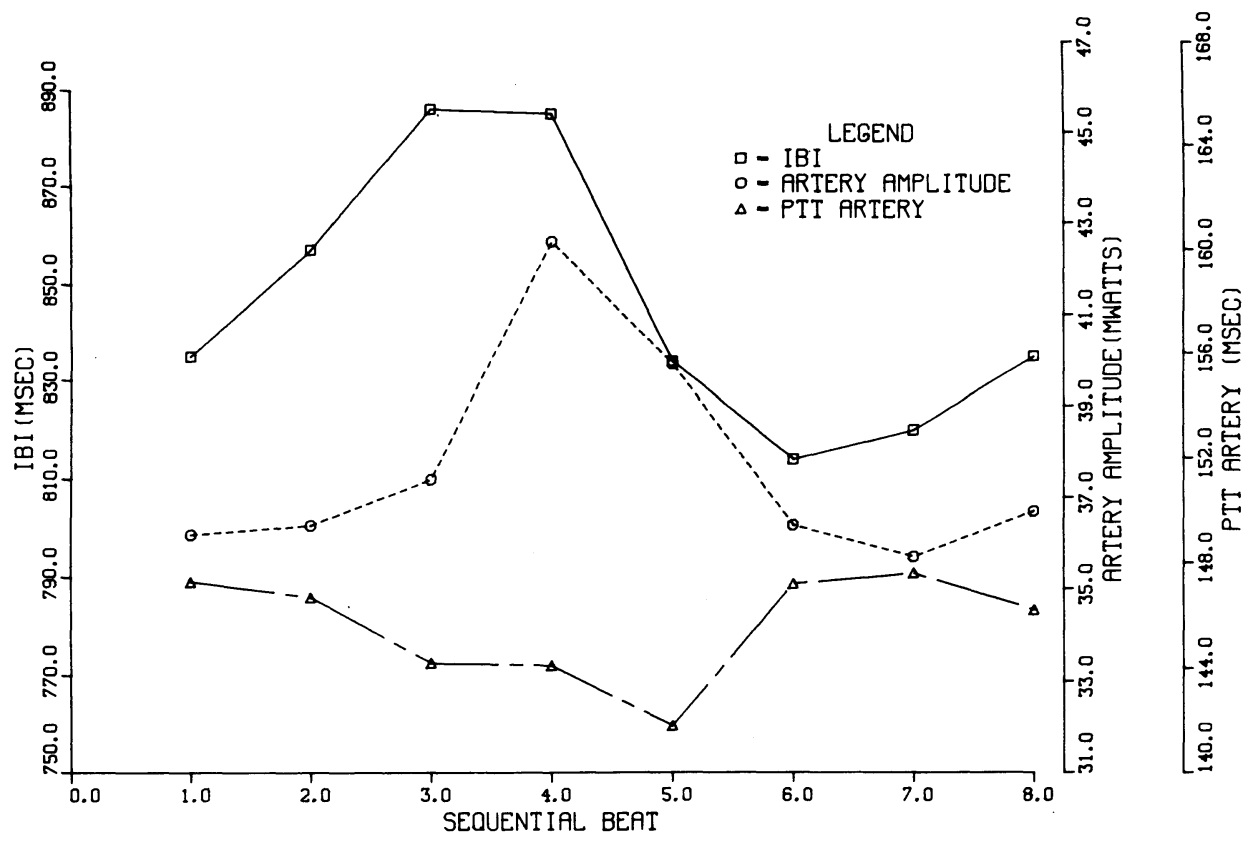

Figure 1. Beat-by-beat responses during performance of the RT task. The stimulus occurs at the end of Beat 3 and thus corresponds in time to the 3.0 beat on the $x$-axis. From top to bottom, interbeat interval, artery pulse amplitude, and $R$ wave to artery PTT are displayed.

same effects were present in the thumb signals [PTT, $F(1,25)=6.3$; amplitude, $F(1,25)=7.8$, adjusted df]. Figure 1 suggests that changes in IBI might be correlated and possibly account directly for the vascular results. Correlations between measures may be assessed across volunteers or trials (within each volunteer). Across-volunteer correlations showed that volunteers with greater slowing of the heart rate (a Beat 4 - Beat 1 difference score) showed a greater shortening of thumb PTT $(r=.44)$. Correlations with arterial and amplitude measures were not significant. Across-trial correlations showed that trial-to-trial variability in deceleration was not directly related to similar variability in PTT. Correlations run withinsubject across trials for a subsample of $\mathbf{3 0}$ sessions showed essentially zero correlation (average $r$ for IBI with thumb PTT $=.04$; with subclavian PTT $=.02$ ).
Incentive and target effects. The effects of target on the cardiac and vascular variables were similar to those reported previously (Jennings, Schrot, \& Wood, 1980). Table 2 presents the relevant means. IBI is presented as a difference score expressing the amount of cardiac deceleration (Beat 4 - Beat 1 ). As expected, deceleration was largest for the targets that induced rapid responding [beat $\times$ target, $F(6,180)=3.35$, adjusted df]. Systolic and diastolic pressures were higher at the end of targets that required faster, as opposed to slower, response [target effects: SBP, $F(1,28)=15.27 ; D B P, F(1,28)=4.44$, adjusted df]. The gradient shown by PWV was similar to that for the pressure measures. Higher velocities were associated in general with faster response quintiles [target, $F(3,36)=3.27$, adjusted df]. Thumb and subclavian amplitudes were not significantly in-

Table 2

Cardiovascular Response to Variation in RT Target

\begin{tabular}{lrrrrr}
\hline & \multicolumn{4}{c}{ Target } \\
\cline { 2 - 5 } & \multicolumn{1}{c}{150} & 200 & 250 & 300 & 350 \\
\hline Cardiac Deceleration (Beat 4-1) & 58 & 59 & 53 & 57 & $50^{*}$ \\
Systolic/Diastolic Pressure & $135 / 81$ & $133 / 81$ & $131 / 80$ & $131 / 80$ & $130 / 80^{*}$ \\
Pulse Wave Velocity & 12.9 & 13.2 & 12.0 & 12.2 & $11.0^{*}$ \\
Thumb Amplitude & 20.7 & 22.8 & 20.8 & 22.1 & 18.5 \\
Subclavian Amplitude & 36.7 & 37.1 & 38.9 & 39.0 & 36.5 \\
\hline
\end{tabular}

Note-Deceleration is given in milliseconds; pressure is given in millimeters of mercury; velocity is given in meters per second; amplitude is given in microwatts. ${ }^{*}$ Statistically significant target effect. 
fluenced by target. The ordering of the thumb amplitudes was, however, in the same direction as that previously found (Jennings, Schrot, \& Wood, 1980).

Incentive altered IBI and arterial PTT but, unlike target, failed to affect PWV or blood pressure. The average IBIs were $826 \mathrm{msec}$ in the low-incentive condition and $865 \mathrm{msec}$ in the high-incentive condition [incentive main effect, $F(1,29)=4.51$ ]. Arterial PTT decreased with high incentive, but only at the time of and immediately after performance. Figure 2 shows this effect [incentive $\times$ beat, $F(5,113)=2.81$, adjusted df]. Increasing the incentive increased the depth of the PTT response to task performance.

Correctness. For the three fastest targets, 150, 200 , and $250 \mathrm{msec}$, errors were frequent. This permitted a meaningful comparison of trials that were correctly and incorrectly (i.e., the wrong RT key was pressed) performed. Cardiovascular data from these target conditions were submitted to an analysis of variance with a correct versus error factor (as well as the beats, incentive, and target factors). The analysis showed a small but significant effect of correctness on pulse transit time. Transit times for both thumb and subclavian signals were about $1 \mathrm{msec}$ longer in the correct trials than in the errors [for thumb, mean difference $=1.2 \mathrm{msec}, F(1,29)=9.55$; for subclavian, mean difference $=.6 \mathrm{msec}, F(1,29)=$ 5.19]. Figure 3 shows that correct error differences for thumb PTT were modified by incentive and tar- get $[F(1,29)=5.88]$. High incentive induced lower PTT for the faster but not for the slower responses and also altered the correct error differences. Analyses of the simple effects showed that correct error differences due to incentive were only significant in the 150-msec target condition [incentive $\times$ target, $F(1,25)=9.67]$. Correct trial PTTs were longer than errors for this target, but only with high-incentive instructions. Neither IBI nor the vascular indices were altered by the correctness of the response.

\section{DISCUSSION}

The primary aim of this experiment was to find out if adaptive vascular changes occurred during anticipatory cardiac deceleration. Furthermore, we wished to separate passive hemodynamic influences from changes induced by sympathetic and parasympathetic neural influences. Brief, event-related or phasic changes are discussed here separately from longer term (tonic) cardiovascular adjustments.

Phasic response during task performance. Both vagal and sympathetic efferents to the heart are tonically active and capable of rapid adjustments (e.g., W. C. Randall, 1977). Typically, vagal control shows the shorter latency, but sympathetic responses are rapid enough to induce changes during the $6 \mathrm{sec}$ or so during which anticipation could develop in the current study (D. C. Randall, 1977). Pharmacolog-

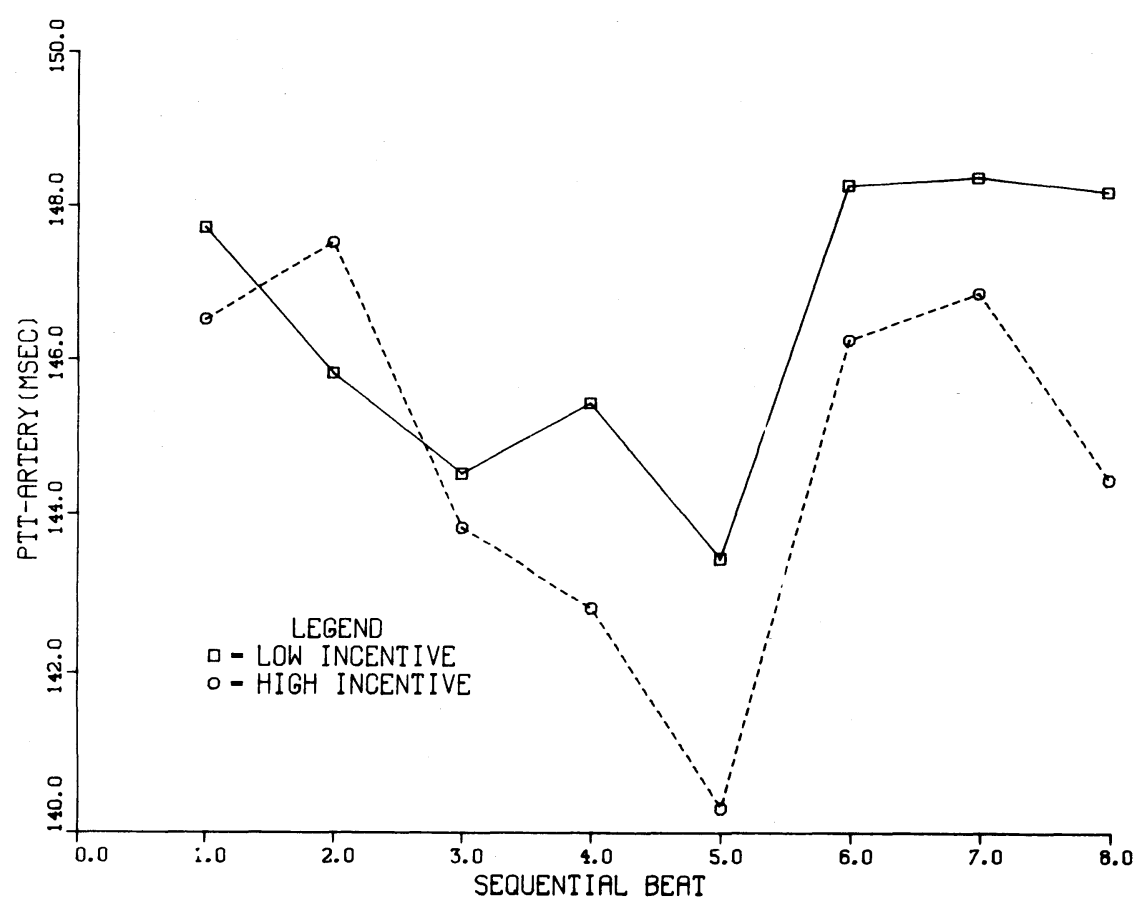

Figure 2. Beat-by-beat change in PTT to the artery site during the performance of the RT task. The stimulus time corresponds to the 3.0 on the $x$-axis. The results for low- and highincentive conditions are presented separately. 


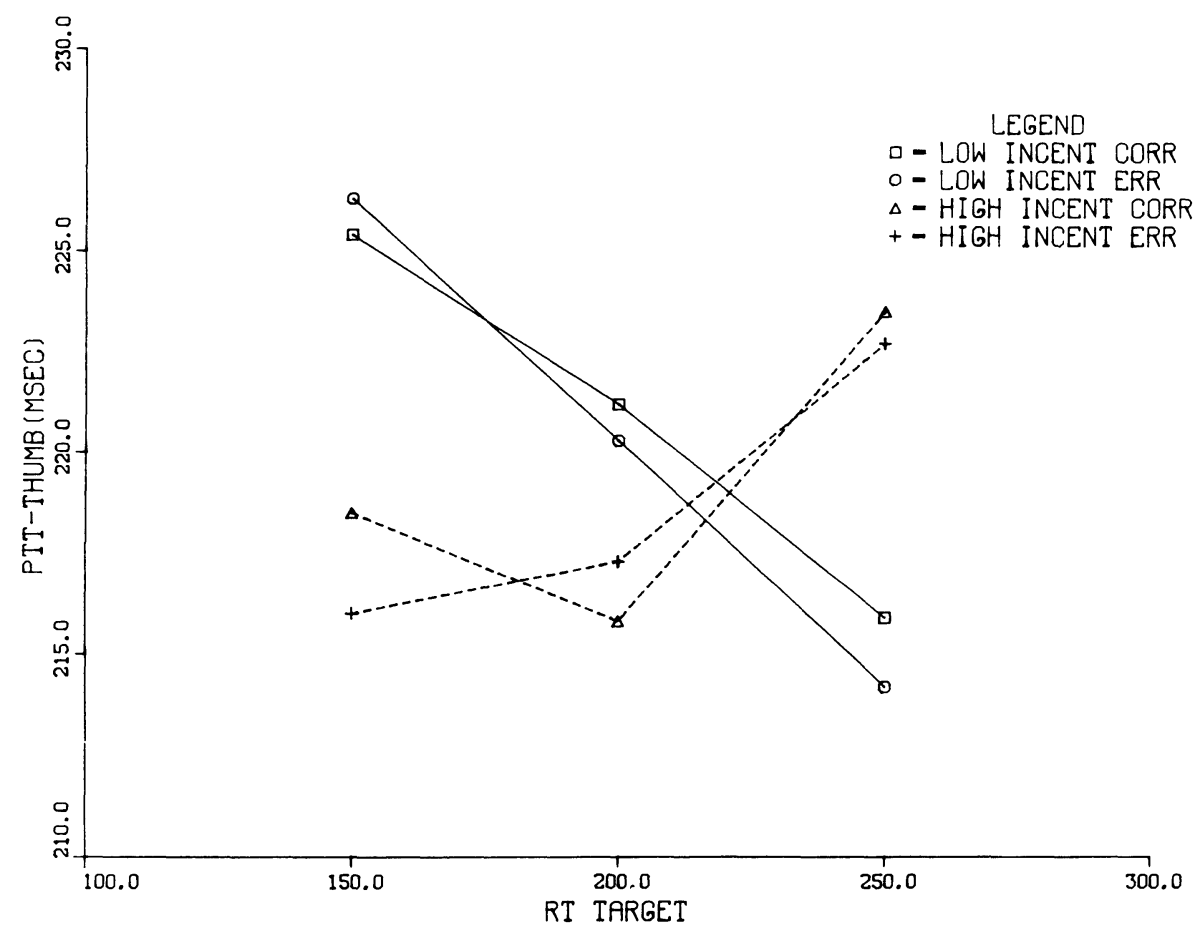

Figure 3. The interactive effects of RT target, incentive, and response correctness on transit time to the thumb site. Post hoc analyses suggested that correct - error differences were significant only for the 150-msec target.

ical studies in humans (see Obrist, 1981) have established that vagal efferents control anticipatory heart rate deceleration.

Our understanding of the vascular changes is less complete. Obrist (1981) and Obrist, Wood, and Perez-Reyes (1956) explored the possibility that anticipatory heart rate deceleration was simply a reflex response to a phasic blood pressure increase. Their results, however, questioned this possibility. Invasive blood pressure readings were elevated minimally during anticipation, and the blocking of vascular effects with an alpha adrenergic agent failed to alter cardiac deceleration. In the current data, alpha adrenergic effects would be most clearly seen in a shortening of arterial-to-peripheral PWV (see Jennings $\&$ Choi, in press). PWV failed to show any phasic change, thus supporting Obrist's (1981) observation of minimal alpha sympathetic change during anticipation. Gliner, Browe, and Horvath (1977), using impedance techniques, reported that anticipatory deceleration was associated with an increase in stroke volume. A derived cardiac index was not significantly altered. Gliner et al.'s (1977) stroke-volume finding suggests beta adrenergic or filling effects and may be relevant to our PTT and amplitude results.

Phasic changes were observed in both thumb and arterial PTTs (see Figure 1). Similar changes were observed by Goldband (1980). These changes can probably be attributed, at least indirectly, to vagal activation. The parallel changes in thumb and subclavian sites suggest that the PTT change may originate centrally in intracardiac timing, that is, in shortening of the preejection period (PEP). The slowing of heart rate, other things being equal, might be expected to increase cardiac filling (cf. Gliner et al., 1977). An increase in filling can induce a shortening of PEP (Lewis, Leighton, Forester, \& Weissler, 1974). Increased filling should also increase cardiac output and thus arterial and peripheral blood flow. The phasic increase in pulse amplitude that we observed confirms this expectation if the enhanced photo signal amplitude is related to flow (see Jennings, Tahmoush, \& Redmond, 1980, and Jennings \& Choi, in press). Gliner et al.'s (1977) stroke-volume result is also supportive, although Gliner et al. do not advocate a cardiac-filling explanation.

In sum, I suggest that the phasic slowing of heart rate may have increased cardiac filling and indirectly (via the Starling effect) shortened PEP. My attempts to directly confirm this relationship met with mixed success. Within-volunteer correlation of IBI and PTT changes were not significant, but acrossvolunteer correlations did show a significant correlation between IBI and thumb PTT change. The results seem strong enough, however, to tentatively favor a vagal, heart rate-mediated explanation for 
the phasic changes in PTT and pulse amplitude. This interpretation is also consistent with the changes in phasic PTT induced by incentive (see Figure 2). High-incentive sessions were characterized by both a slowed heart rate and a phasically shortened PTT. A viable but more complicated explanation of the phasic changes would be the joint activation of cardiac vagal and beta adrenergic effectors. Vagal chronotropic effects are thought to dominate sympathetic effects (Levy, 1977; Levy \& Martin, 1979). The opposite may occur for inotropic effects. Thus, joint vagal-sympathetic action might yield the observed effects.

Tonic effects. During the performance of the task at different difficulty levels, psychophysiological adjustments may support maintained performance as difficulty increases (Jennings, in press-a, in press-b; Kahneman, 1973). These adjustments may last over a whole period of performance, as opposed to the phasic task-synchronized adjustments discussed previously. Obrist (1981) has previously emphasized the importance of the sympathetic nervous system in these tonic, but task-related, adjustments. His work has shown an increase in beta sympathetic tone during tasks that require "active coping." My tasks were similar, but the measurements were focused on vascular, alpha sympathetic, rather than beta sympathetic, changes. The results showed that PWV and blood pressure were higher during the targets that required relatively faster responses. In Jennings, Schrot, and Wood (1980) and Jennings and Wood (1977), volunteers rated these targets as requiring relatively more attention or effort. Increased effort is consistent with enhanced alpha sympathetic tone for these target conditions. Our data do not speak directly to the question of beta sympathetic change. Vagal activity as indicated by phasic deceleration is stronger during the same targets showing alpha sympathetic effects. Concomitant beta sympathetic effects would be seen most clearly in changes in PEP or cardiac contractility. These measures are not available, but PTT, a variable related to PEP, was not consistently changed by target condition.

Our previous work (Jennings, Schrot, \& Wood, 1980) has shown thumb pulse amplitude changes as a function of target-greater amplitudes being associated with faster targets. The current amplitude results show only a trend in the same direction. Our initial result was interpreted as an inhibition of sympathetic vascular tone. Given our current PWV results, this interpretation appears incorrect. Measurement errors may account for the differences, but it may also be that the task induces both alpha and beta sympathetic responses, so that beta-induced contractility changes are posed against alpha-induced changes in vascular caliber and compliance. This interpretation is speculative, however, particularly in the light of our failure to show target effects in subclavian PTT.

The current results are reasonably clear in suggesting concomitant vagal effects on the heart and alpha sympathetic effects on the vasculature. The form of autonomic patterning is no longer surprising, although it contradicts early arousal notions (e.g., Lindsley, 1960). Lacey et al. (1963) showed some time ago that skin conductance activation could covary with cardiac deceleration. Our work shows a conceptually similar patterning in the cardiovascular system. Neurophysiological work has also shown that autonomic responses are patterned, rather than being solely sympathetic or parasympathetic. For example, Koizumi, Yamashita, Kollai, and Brooks (1979) showed that chemoreceptor stimulation produced cardiac vagal excitation, excitation of sympathetic vasoconstriction in muscle, and also inhibition of cardiac sympathetic activity.

Correct - error differences. The most interesting results from a psychological perspective are those for correct trials compared with error trials. Errors in buttonpressing occurred intermittently and were probably related to brief changes in alertness or effort. The observed physiological differences, however, were not specifically localized prior to or after the stimulus. Thus, they are probably not related to degree of anticipation (prior to the stimulus) or to affective reactions to error (after the stimulus).

Pulse transit time but not PWV or IBI was influenced by correctness. I have suggested that PTT changes in the absence of PWV differences may indicate effects on PEP. PEP is altered by cardiac sympathetic activity as well as by the indirect hemodynamic effects discussed above (W. C. Randall, 1977). My results showed that PTT was longer during correct trials. This effect is consistent with relatively less cardiac sympathetic effects on correct trials. Reports from volunteers suggest that many errors are due to the failure to inhibit a proposed response when the opposite stimulus occurs. Heightened sympathetic activity may be incompatible with the inhibition described. Some support for this speculation may be found in the thumb PTT results, which are altered by incentive and quintile of RT as well (see Figure 3). This figure shows that high but not low incentive induced short PTTs for the targets that required rapid responses. Furthermore, for the 150msec target, the heightened cardiac sympathetic activity led to a reversal of correct - error PTTsPTTs for correct responses were longer in the highincentive condition and marginally shorter in the low-incentive condition. The remaining correct error differences shown in Figure 3 failed to be statistically significant. Interpretively, speeded, correct responses may require a rather specific amount of sympathetic support-failure of inhibition will occur 
if too much is provided, and speed may suffer if too little is provided. Admittedly, the differences shown are small, and much more work is needed to establish whether PTT (or, more ideally, PEP) measures are indeed sensitive to the success and failure of response inhibition.

Cardiovascular response patterning and its significance. The current results support our previous conclusion (Jennings, Schrot, \& Wood, 1980) that performance tasks can induce combined sympathetic and parasympathetic effects. Vagal cardiac effects can occur in the context of vascular sympathetic effects. As in the Jennings, Schrot, and Wood (1980) paper, we suggested that the vagal effects were predominantly brief beat-by-beat effects combined with somewhat more sustained sympathetic effects. The results suggested that the brief vagal effects might indirectly facilitate stroke volume in support of anticipated action. Change in vascular and, inferentially, in cardiac sympathetic activity was related to response-speed conditions. This relationship, as well as small but significant correct - error differences, suggested that sympathetic excitation might be related to the threshold of response initiation.

I have attempted to indirectly assess cardiovascular responses that can be interpreted in terms of specific autonomic response patterns. The results are suggestive, but the techniques used are relatively new. The interpretations must remain tentative until replication combined with pharmacological and convergent measure approaches can verify them.

\section{REFERENCES}

Burton, A. C. Physiology and biophysics of the circulation (2nd ed.). Chicago: Yearbook Publishers, 1972.

Coote, J. H., Hilton, S. M., \& Perez-Gonzalez, J. F. Inhibition of the baroreceptor reflex on stimulation in the brain stem defence centre. Journal of Physiology (London), 1979, 288, $549-560$.

Djojosugito, A. M., Folkow, B., KYlstra, P. H., Lisander, B. S., \& Tutrue, R. S. Differentiated interaction between the hypothalamic defence reaction and baroreceptor reflexes. I. Effects on heart rate and regional flow resistance. Acta Physiologica Scandinavica, 1970, 78, 376-385.

Gliner, J. A., Browe, A. C., \& Horvath, S. M. Hemodynamic changes as a function of classical aversive conditioning in human subjects. Psychophysiology, 1977, 14, 281-286.

GoldBAND, S. Environmental specificity of physiological response to stress in coronary-prone subjects. Journal of Personality and Social Psychology, 1980, 39, 670-679.

Jenninas, J. R. Bodily changes during attending. In M. G. H. Coles, E. Donchin, \& S. W. Porges (Eds.), Psychophysiology: Systems processes, and applications. New York: Guilford, in press. (a)

Jennings, J. R. Memory, thought, and bodily response. In M. G. H. Coles, E. Donchin, \& S. W. Porges (Eds.), Psychophysiology: Systems, processes, and applications. New York: Guilford, in press. (b)

Jennings, J. R., \& Choi, S. Use of an optical sensor for arterial to peripheral pulse wave velocity measures. Psychophysiology, in press.
Jennings, J. R., Schrot, J., \& Wood, C. C. Cardiovascular response patterns during choice reaction time. Physiological Psychology, 1980, 8, 130-136.

Jennings, J. R., Tahmoush, A. J., \& Redmond, D. Peripheral vascular measurement. In P. H. Venables \& I. Martin (Eds.), Techniques in psychophysiology. London: Wiley, 1980.

Jennings, J. R., \& Wood, C. C. The E-adjustment procedure for repeated measures analyses of variance. Psychophysiology, 1976, 13, 277-278.

Jennings, J. R., \& Wood, C. C. Cardiac cycle time effects on performance, phasic cardiac responses, and their intercorrelation in choice reaction time. Psychophysiology, 1977, 14, 297-307.

Kahneman, D. Attention and effort. Englewood Cliffs, N.J: Prentice-Hall, 1973.

Koizumi, K., Yamashita, H., Kollai, M., \& Brooks, C. McC. Differential responses in reflex action: The question of blood volume and pressure control. In C. McC. Brooks, K. Koizumi, \& A. Sato (Eds.), Integrative functions of the autonomic nervous system. Tokyo: University of Tokyo Press, 1979.

LACEY, B. C., \& LACEY, J. I. Studies of heart rate and other bodily processes in sensorimotor behavior. In P. A. Obrist, A. H. Black, \& L. V. DiCara (Eds.), Cardiovascular psychophysiology. Current issues in response mechanisms, biofeedback, and methodology. Chicago: Aldine-Atherton, 1974.

LACEY, B. C., \& LACEY, J. I. Two way communication between the heart and the brain. American Psychologist, 1978, 33, 99-113.

LACEY, J. I. Some cardiovascular correlates of sensorimotor behavior: Example of visceral afferent feedback? In C. H. Hockman (Ed.), Limbic system mechanisms and autonomic function. Springfield, Ill: Thomas, 1972.

Lacey, J. I., Kagan, J. R., Lacey, B. C., \& Moss, H. A. The visceral level: Situational determinants and behavioral correlates of autonomic response patterns. In P. H. Knapp (Ed.), Expression of the emotions in man. New York: International University Press, 1963.

Levy, M N. Parasympathetic control of the heart. In W. C. Randall (Ed.), Neural regulation of the heart. New York: Oxford University Press, 1977.

LEvy, M. N., \& MARTin, P. J. Neural control of the heart. In R. M. Berne, N. Sperelakis, \& S. R. Geiger (Eds.), Handbook of physiology. Section 2: The cardiovascular system. Bethesda, Md: American Physiological Society, 1979.

Lewis, R., Leighton, R., Forestor, W., \& Weissler, A. Systolic time intervals. In A. Weissler (Ed.), Noninvasive cardiology. New York: Grune \& Stratton, 1974.

Light, K. C., \& Oвrist, P. A. Cardiovascular response to stress: Effects of opportunity to avoid shock experience and performance feedback. Psychophysiology, 1980, 17, 243-252.

LindsLex, D. B. Attention, consciousness, sleep, and wakefulness. In H. W. Magoun (Ed.), Handbook of physiology. Section 1: Neurophysiology (Vol. 3). Washington, D.C: American Physiological Society, 1960.

Manuck, S. B., \& GARLAND, F. N. Coronary-prone behavior pattern, task incentive, and cardiovascular response. Psychophysiology, 1979, 16, 136-142.

Oвrist, P. A. Cardiovascular psychophysiology: A perspective. New York: Plenum, 1981.

Obrist, P. A., Howard, J. L., Lawler, J. E., Galosy, R. A., Meyers, K. A., \& Gaebelein, C. J. The cardiac-somatic interaction. In P. A. Obrist, A. H. Black, J. Brener, \& L. V. DiCara (Eds.), Cardiovascular psychophysiology: Current issues in responses mechanisms, biofeedback, and methodology. Chicago: Aldine, 1974.

Obrist, P. A., Webb, R. A., Sutterer, J. R., \& Howard, J. L. Cardiac deceleration and reaction time: An evaluation of two hypotheses. Psychophysiology, 1970, 6, 695-706.

Obrist, P. A., Wood, D. M., \& Perez-Reyes, M. Heart rate during conditioning in humans: Effects of UCS intensity, vagal 
blockade, and andrenergic block of vasomotor activity. Journal of Experimental Psychology, 1965, 70, 32-42.

Randall, D. C. Neural control of the heart in the intact nonhuman primate. In W. C. Randall (Ed.), Neural regulation of the heart. New York: Oxford University Press, 1977.

RANDAll, W. C. (Ed.). Neural regulation of the heart rate. New York: Oxford University Press, 1977.

Shimizu, H. Reliable and precise identification of R-waves in the EKG with a simple peak detector. Psychophysiology, 1978, 15, 499-501.

Williams, R. B., Jr., Bittker, T. E., Buchsbaum, M. S.,
\& Wynne, L. Cardiovascular and neurophysiological correlates of sensory intake and rejection. I. Effect of cognitive tasks. Psychophysiology, 1975, 12, 427-433.

Wood, C. C., \& Jennings, J. R. Speed-accuracy tradeoff functions in choice reaction time: Experimental designs and computation procedures. Perception \& Psychophysics, 1976, 19, 92-102.

(Manuscript received July 6, 1982; revision accepted for publication December 2, 1982.) 